\title{
Smoothened inhibition leads to decreased cell proliferation and suppressed tissue fibrosis in the development of benign prostatic hyperplasia
}

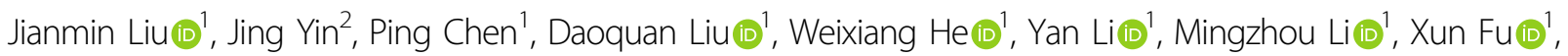

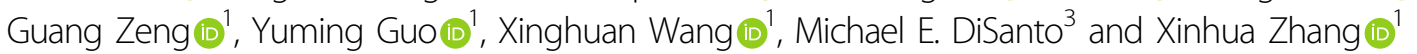

\begin{abstract}
Benign prostatic hyperplasia (BPH) is a common disease in aging males. It has been proven that the Hedgehog $(\mathrm{HH})$ is implied as an effective and fundamental regulatory growth factor signal for organogenesis, homeostasis, and regeneration. Smoothened (SMO), as the major control point of HH signals, activates aberrantly in most human solid tumors. However, the specific function of SMO and its downstream glioma-associated oncogene (GLI) family in BPH has not been well understood. Here, we first revealed that the SMO cascade was upregulated in BPH tissues and was localized in both the stromal and the epithelium compartments of human prostate tissues. Cyclopamine, as a specific SMO inhibitor, was incubated with BPH-1 and WPMY-1, and intraperitoneally injected into a BPH rat model established by castration with testosterone supplementation. SMO inhibition could induce cell apoptosis, cell cycle arrest at the G0/G1 phase, and a reduction of tissue fibrosis markers, both in vitro and in vivo. Finally, a tissue microarray, containing 104 BPH specimens, was constructed to analyze the correlations between the expression of SMO cascade and clinical parameters. The GLI2 was correlated positively with nocturia and negatively with fPSA. The GLI3 was in a positive relationship with International Prostate Symptom Score and nocturia. In conclusion, our study suggested that SMO cascade could play important roles in the development of $\mathrm{BPH}$ and it might be rediscovered as a promising therapeutic target for $\mathrm{BPH}$.
\end{abstract}

\section{Introduction}

Benign prostatic hyperplasia (BPH) refers to the nonmalignant growth of the prostate that occurred commonly in aging men and can lead to lower urinary tract symptoms (LUTS) ${ }^{1}$. Bothersome LUTS can interfere with activities of daily living, causing significant impairment of disease-specific quality of life. The presence of histological $\mathrm{BPH}$ at autopsy is $\sim 50 \%$ in those aged $51-60$ years and $70 \%$ in those aged $61-70$ years $^{2}$. Androgen levels and

\footnotetext{
Correspondence: Xinhua Zhang (zhangxinhuad@163.com)

${ }^{1}$ Department of Urology, Zhongnan Hospital of Wuhan University, Wuhan,

China

2Department of Rehabilitation, Zhongnan Hospital of Wuhan University,

Wuhan, China

Full list of author information is available at the end of the article

These authors contributed equally: Jianmin Liu, Jing Yin, Ping Chen,

Daoquan Liu

Edited by I. Amelio
}

aging are two indispensable driving factors of $\mathrm{BPH}^{3}$. Despite the high prevalence, the causative factors of the initial event leading to the development of $\mathrm{BPH}$ remain unclear. Consequently, it is of great significance to identify new markers and new therapeutic targets for $\mathrm{BPH}$.

It has been documented that robust Hedgehog $(\mathrm{HH})$ signals occur frequently during embryonic development and infancy ${ }^{4}$, playing an instrumental role during diverse processes of cell differentiation and organ development (e.g., regulating cavernosa and chondrocyte differentiation $^{5,6}$, coordinating lung specification, branching morphogenesis, and foregut mesenchymal differentiation ${ }^{7}$ ). New glands, which can only be seen in the embryonic period, are often found in the human hyperplastic prostate. It has been proposed that the occurrence of BPH is the "reawakening" of the embryonic process that prostate mesenchyme induced epithelial differentiation ${ }^{8}$. Several

\section{(c) The Author(s) 2021}

(c) Open Access This article is licensed under a Creative Commons Attribution 4.0 International License, which permits use, sharing, adaptation, distribution and reproduction in any medium or format, as long as you give appropriate credit to the original author(s) and the source, provide a link to the Creative Commons license, and indicate if changes were made. The images or other third party material in this article are included in the article's Creative Commons license, unless indicated otherwise in a credit line to the material. If material is not included in the article's Creative Commons license and your intended use is not permitted by statutory regulation or exceeds the permitted use, you will need to obtain permission directly from the copyright holder. To view a copy of this license, visit http://creativecommons.org/licenses/by/4.0/. 
$\mathrm{HH}$ target genes have been identified in the mesenchyme of developing prostate, including the cytokine Cxcl14, the insulin-like growth factor-binding protein $I g f b p 3$, and the delta/notch-like epidermal growth factor-related receptor Dner ${ }^{9}$. Indeed, HH pathway target genes have been implicated in the placement of prostate epithelial buds and in subsequent ductal branching and outgrowth ${ }^{10}$.

The activation of $\mathrm{HH}$ signaling cascade is initiated by binding $\mathrm{HH}$ ligands to the membrane receptor Patched1 (PTCH1), thus releasing smoothened (SMO) to be activated by phosphorylation. SMO ultimately decreases the interaction between suppressor of fused homolog and gliomaassociated oncogenes (GLIs). GLI proteins belong to zincfinger transcription factors and are the terminal effectors of SMO cascade. This family consists of three major regulators in mammals, i.e., GLI1-3. GLI1 acts only as a transcriptional activator (GLI-A), whereas GLI2 and GLI3 can be processed into both transcriptional activators and repressors (GLI-R), depending on the specific cell context and on the activation state of $\mathrm{HH}$ signaling ${ }^{11}$.

The transmembrane protein SMO, a member of the Frizzled class of G-protein-coupled receptor superfamily, is a critical control point of the HH signaling pathway. The accumulation of activated SMO suppresses the generation of GLI-R forms and allows GLI-A proteins to translocate into the nucleus and transcriptionally activate their target genes that include HH-GLI pathway autoregulation (GLI1, PTCH1, HHIP1), proliferation and differentiation (e.g., cyclin D1, cyclin E, E2F1, FOXM1, MYC, IGFBP3), apoptosis (e.g., $B c l-2)$, epithelial-to-mesenchymal transition (e.g., SNAIL, MMP9, ZEB1, ZEB2), and stem cell self-renewal (e.g., NANOG, SOX2) ${ }^{12,13}$. Contrarily, in the absence of $\mathrm{HH}$ ligand, PTCH1 represses the $\mathrm{HH}$ signals by inhibiting the activity of SMO. Therefore, SMO is the central transductor in $\mathrm{HH}$ signaling and is of major academic and pharmaceutical interest. In addition, the actions of $\mathrm{HH}$ signaling, especially SMO and its downstream GLI family, on growth, epithelial-mesenchymal interactions, and fibrosis in $\mathrm{BPH}$ are not well documented.

The current study concentrated on SMO and its downstream GLI family of proteins in the regulation of $\mathrm{BPH}$ development. Our study extended the functional activities of SMO to human prostate tissues and human prostate cell lines. Furthermore, in vivo effect of SMO cascade was investigated with SMO inhibitor $\mathrm{CYC}$ treating $\mathrm{BPH}$ rat model established by castration with testosterone supplementation.

\section{Results}

The expression and localization of SMO and GLI1-3 in human prostate tissues and cell lines

Three Gene Expression Omnibus (GEO) datasets (GSE6099, GSE3868, and GSE119195) and the Oncomine database were explored to analyze the differentially expressed SMO cascade genes between BPH specimens and normal prostate (NP) specimens. As shown in Fig. 1A, the expression of SMO and GLI2 at transcriptional levels was significantly upregulated in $\mathrm{BPH}$ when compared with NP through GSE119195 dataset. The messenger RNA (mRNA) expression of GLI2 and GLI3 was significantly increased in BPH observed in Tomlins Prostate $^{14}$ from the Oncomine database (Fig. 1B). Normal and hyperplastic prostate were harvested $(n=16$, eight control samples and eight BPH samples) from our institute. In the hyperplastic prostate, the mRNA level of SMO and GLI1-3 was significantly increased over 2-fold and the protein level of these molecules was consistently increased (Fig. 1C, D). Meanwhile, the immunofluorescence staining showed that SMO and GLI1-3 were localized in both the stromal and the epithelium compartments of the human prostate (Fig. 2A-D). Consistently, they were expressed in epithelium cell $\mathrm{BPH}-1$ and stromal cell WPMY-1 (Fig. 2E-H). Hence, epithelial and stromal cells were used in our subsequent studies.

\section{Human prostate cells with CYC treatment increased cell apoptosis and induced G0/G1 arrest}

Human cell lines were treated with the SMO inhibitor CYC. The Cell Counting Kit-8 (CCK-8) assay was applied to detect $\mathrm{BPH}-1$ and WPMY-1 cell viability after CYC treatment and calculated the half-maximal inhibitory concentration (IC50) of CYC for the cytotoxic effect. We found that the IC50 for the cytotoxic effect of BPH-1 and WPMY-1 cells was 29.8 and $32.6 \mu \mathrm{M}$, respectively (Fig. 3A). Based on the IC50, 5, 10, and $20 \mu \mathrm{M}$ dosages of CYC were chosen. As shown in Fig. 3B, C, SMO and GLI1-3 were dose-dependently downregulated at the transcriptional and translational level in BPH-1 cells. For GLI2 and GLI3, it was more obviously inhibited in WPMY-1 cells, especially at a high dosage of CYC. Moreover, the CCK-8 assay showed that CYC dose-dependently inhibited $\mathrm{BPH}-1$ and WPMY-1 cell viability with a significant difference observed at 48 and $72 \mathrm{~h}$ (Fig. 3D). Meanwhile, cell fluorescence staining revealed that $\mathrm{Ki}-67$-positive cells per field decreased significantly at 10 and $20 \mu \mathrm{M}$ (Fig. 3E, F). In addition, the flow cytometry analysis showed a significant increase in the percentage of apoptotic human epithelial cells by $20.24 \%$ and $45.81 \%$ at 10 and $20 \mu \mathrm{M}$, respectively. CYC treatment also resulted in a significant increase of the percentage of apoptotic human stromal cells by $10.72 \%, 17.34 \%$, and $29.00 \%$ at 5,10 , and $20 \mu \mathrm{M}$ (Fig. $4 \mathrm{~A}$, $\mathrm{B})$, respectively. Furthermore, the flow cytometry analysis also showed that $20 \mu \mathrm{M}$ of CYC led to an increase of the G0/G1 phase cells and a reduction of the G2/M phase cells by $14.58 \%$ and $12.73 \%$ for $\mathrm{BPH}-1$ cells, while a significant increase of the G0/G1 phase cells and a decrease of the G2/M phase cells by $9.37 \%$ and $6.84 \%$ at $20 \mu \mathrm{M}$ for WPMY-1 cells, respectively (Fig. 4C, D). The alterations of proteins associated with cell apoptosis and cell cycle 
A

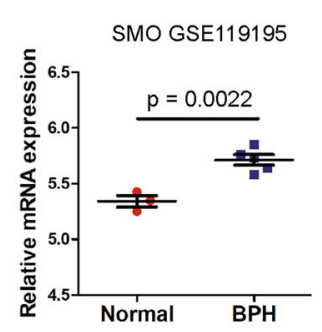

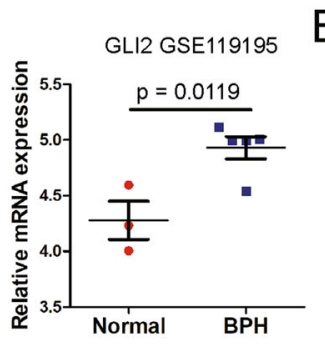
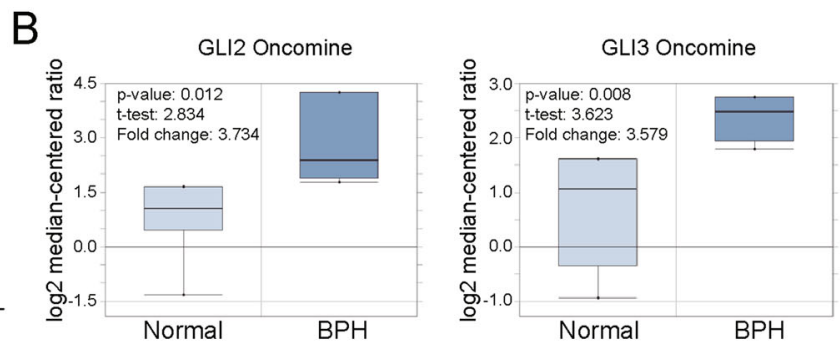

C
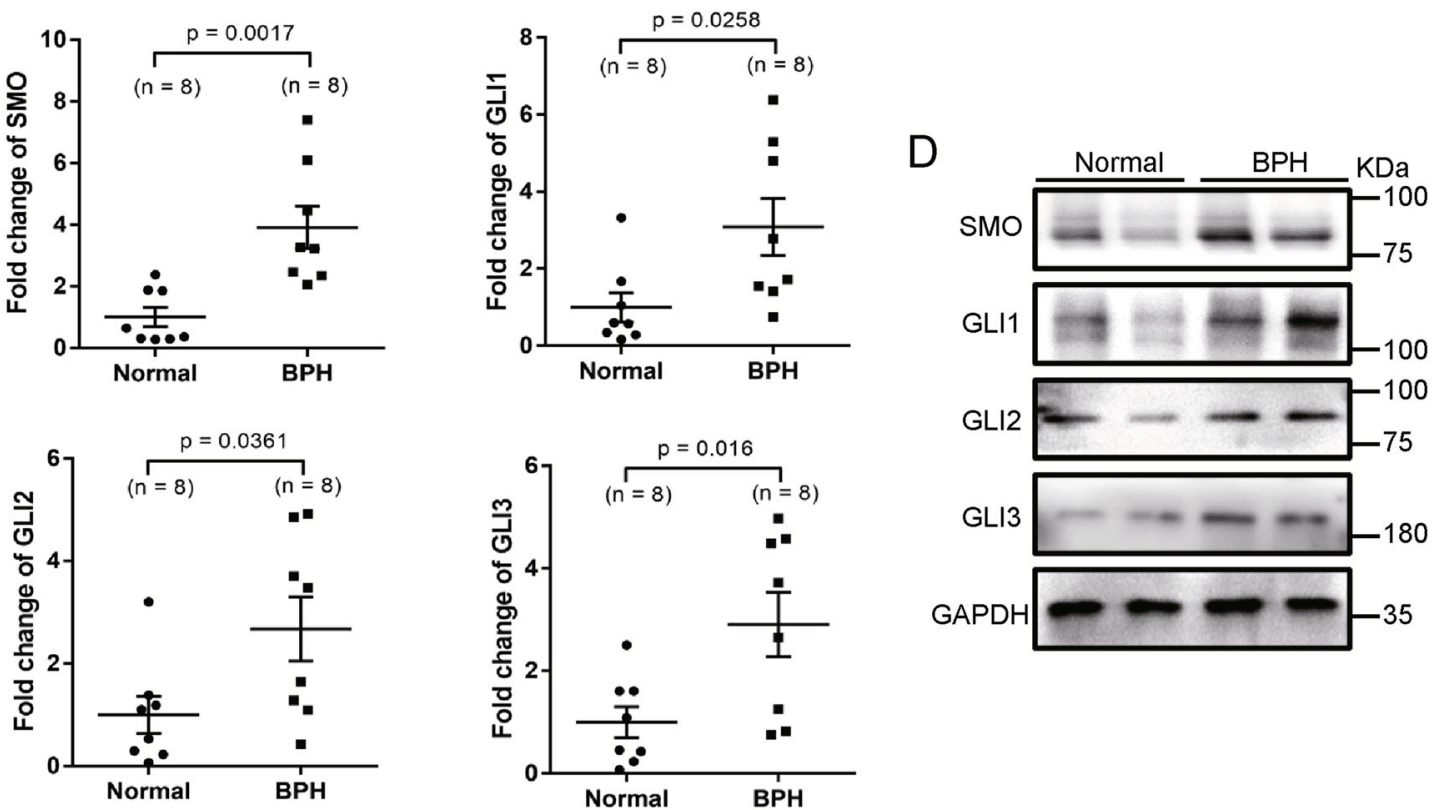

Fig. 1 SMO and GLI1-3 are strongly upregulated in BPH tissues compared with the normal ones. A The mRNA expression of SMO and GLI2 in BPH and NP through GSE119195 dataset. B The mRNA expression of GLI2 and GLI3 in BPH and NP through Tomlins Prostate from Oncomine database. C, D The relative mRNA and protein expression of SMO, GLI1, GLI2, and GLI3 in BPH tissues versus normal prostate tissues. Two-tailed Student's $t$ test.

were further analyzed and the upregulation of the apoptosis inducer BAX and downregulation of a suppressor of apoptosis Bcl-2 in the CYC-treated BPH-1 and WPMY-1 cells were detected. Meanwhile, caspase-3, a downstream protein of $\mathrm{Bcl}-2$ and $\mathrm{BAX}$ in the apoptotic cascade, was decreased, with its active form cleaved caspase-3 increased. Cyclin D1, CDK2, and CDK4 were all decreased in the CYC-treated BPH-1 and WPMY-1 cells, with more obviously at $20 \mu \mathrm{M}$ of CYC (Fig. 4E).

\section{CYC treatment induced a reduction of fibrosis markers in WPMY-1 cells}

As shown in Fig. $5 \mathrm{~A}, \mathrm{~B}$, the fibrosis markers $\alpha$-smooth muscle actin ( $\alpha$-SMA) and collagen I were downregulatedat both mRNA and protein levels in the CYC-treated WPMY1 cells. Similarly, the fluorescence intensity of $\alpha$-SMA and collagen I was decreased in the CYC-treated WPMY-1 cells (Fig. 5 C).

\section{CYC may suppress BPH via decreasing SMO and GLI1-3} in vivo

The in vivo effect of CYC treatment was investigated in rat ventral prostates. The weight of both androgensensitive organs (the ventral prostate and seminal vesicles) was obviously increased for castrated rats with 4-week $\mathrm{T}$ supplementation (BPH model) (Fig. 6A and Supplementary Table S5). Interestingly, the $\mathrm{BPH}$ rats had a significant reduction in body weight during this experimental period (Supplementary Table S5, $p<0.01$ ), which may be due to the physiological effect of $T$. In addition, the ventral prostate weight and prostate index [prostate wet weight (mg)/body weight (g)] were decreased by $22 \%$ and $13 \%$ in CYC-treated rats (Fig. 6A and Supplementary Table S5), respectively $(p<0.05)$. However, the increased weight of the body and seminal vesicles in CYC-treated rats did not reach statistical significance. Histologically, in $\mathrm{BPH}$ rats, the epithelium 


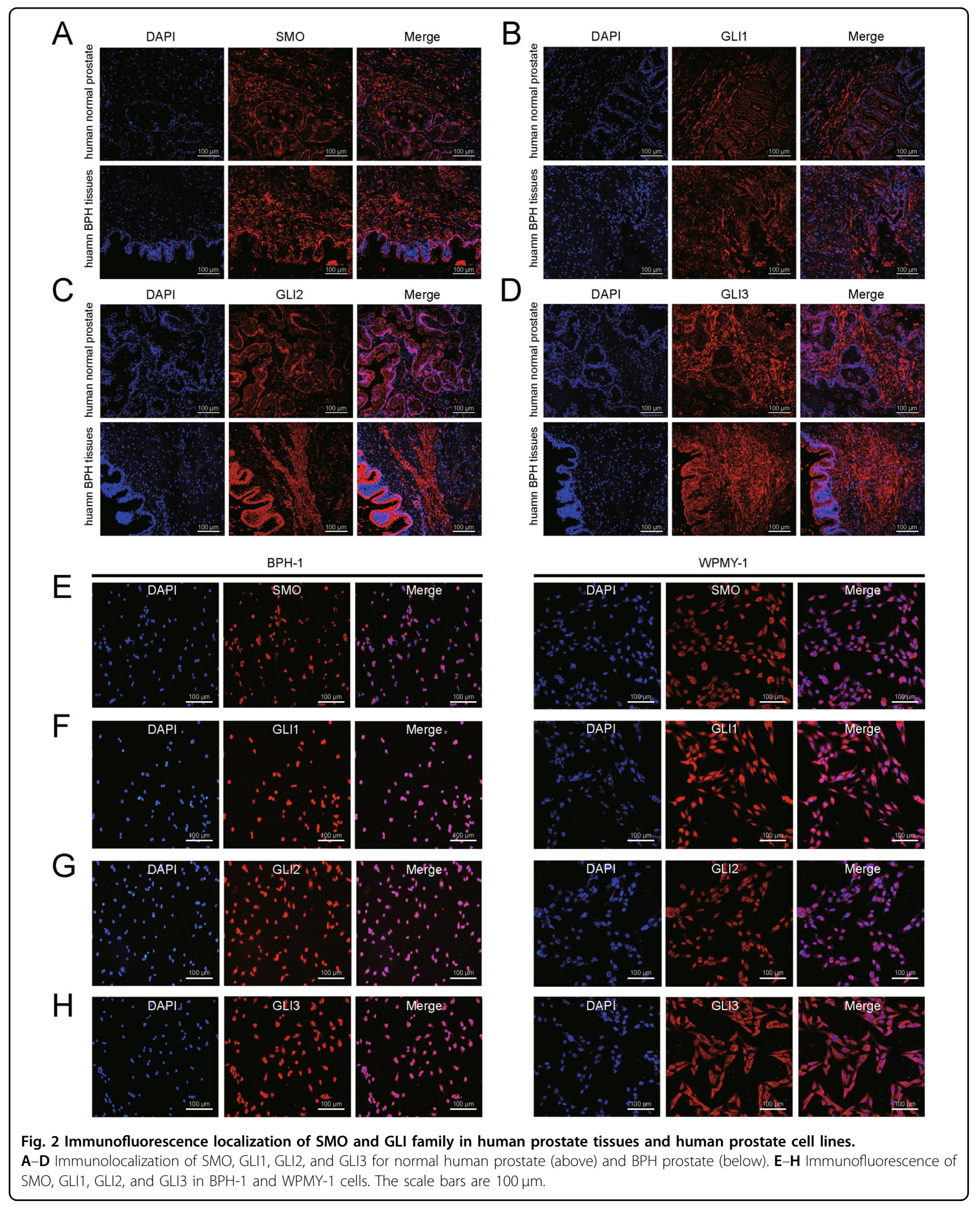



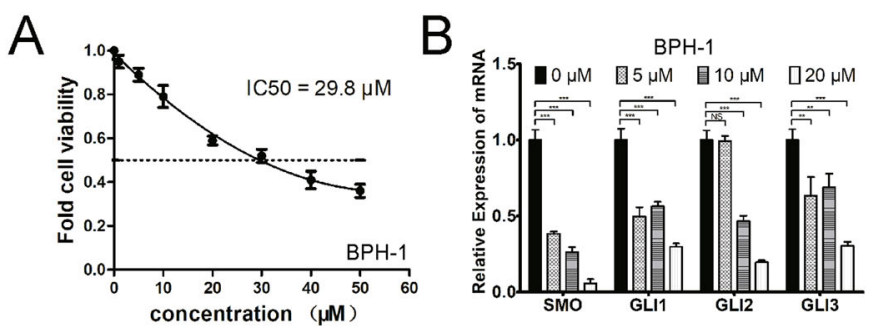

C
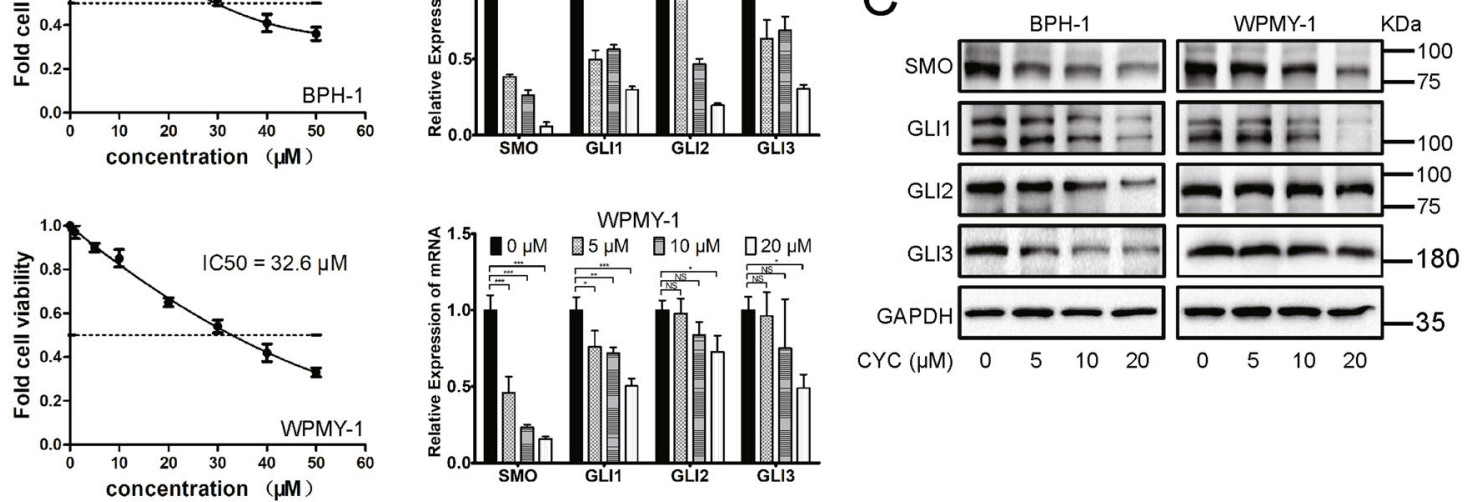

$\mathrm{D}$
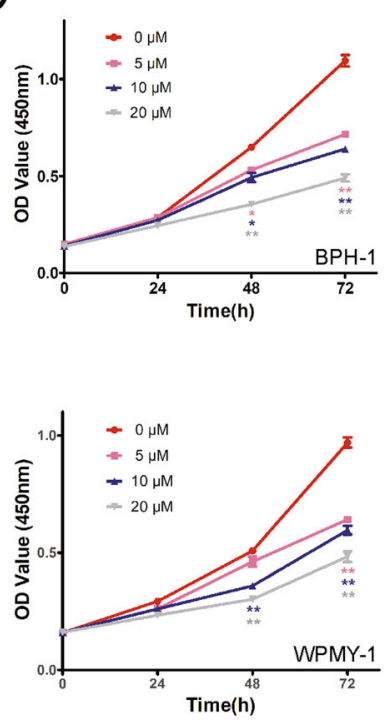

$\mathrm{E}$
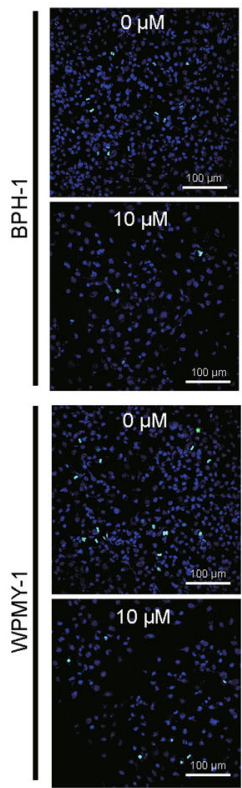
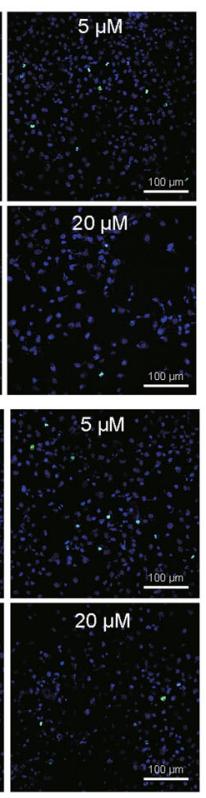

$\mathrm{F}$
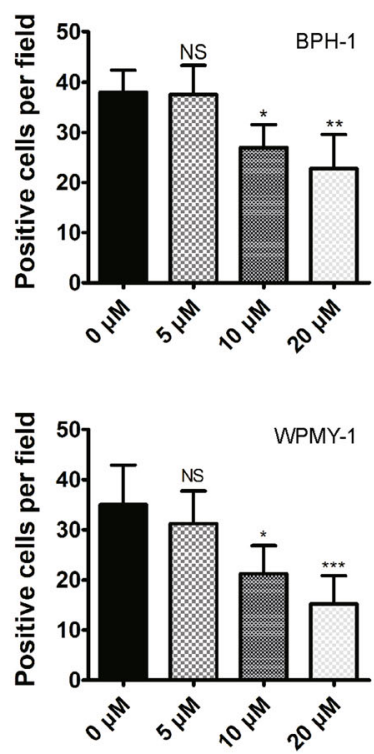

Fig. 3 Typical dose-response curves for the inhibition of proliferation by CYC in BPH-1 and WPMY-1 cells. A The inhibition of proliferation by CYC from 0 to $50 \mu \mathrm{M}$, the IC50 for the cytotoxic effect of BPH-1 and WPMY-1 cells is 29.8 and $32.6 \mu \mathrm{M}$, respectively. B, C The relative mRNA and protein level of SMO, GLI1, GLI2, and GLI3 in BPH-1 and WPMY-1 after CYC treated. D The cell viability of BPH-1 and WPMY-1 after CYC treated at different time points by CCK-8 assay. E The Ki-67 staining of BPH-1 and WPMY-1 after CYC treated. F Statistical analysis of Ki-67-positive cells per field in BPH-1 and WPMY-1 after CYC treated. For 5, 10, and $20 \mu \mathrm{M}$ CYC compared with $0 \mu \mathrm{M} \mathrm{CYC,} \mathrm{NS} \mathrm{means} \mathrm{no} \mathrm{significant} \mathrm{difference,}{ }^{*} p<0.05$, ${ }^{* *} p<0.01$, ${ }^{* * *} p<0.001$; one-way ANOVA. The scale bars are $100 \mu \mathrm{m}$.

component was relatively increased, large glands lined with tall columnar epithelium, which are stratified, pseudostratified, or papillary fronds protruding into the lumen. Nevertheless, SMO blockade by CYC effectively prevented the progression of BPH induced by castration with TP supplementation. The shrunk glands were lined with a single layer of columnar epithelium to low cuboidal cells, along with slight edema (Fig. 6B). Masson's trichrome staining further showed that the hyperplasia of the prostate occurred mainly in the epithelium (increased by 1.3 -fold, $p<0.05$, whereas the stromal component had no difference (either collagen fibers or SM)) in BPH rats. Interestingly, the stromal component was relatively decreased (a $41 \%$ loss of SM and a 50\% loss of collagen fibers) and the epithelium component was also reduced by $26 \%$ in the CYC-treated BPH rat model (Fig. 6C, D). In addition, $\mathrm{CYC}$ treatment downregulated the protein level of SMO and GLI1-3 (Fig. 6E). 

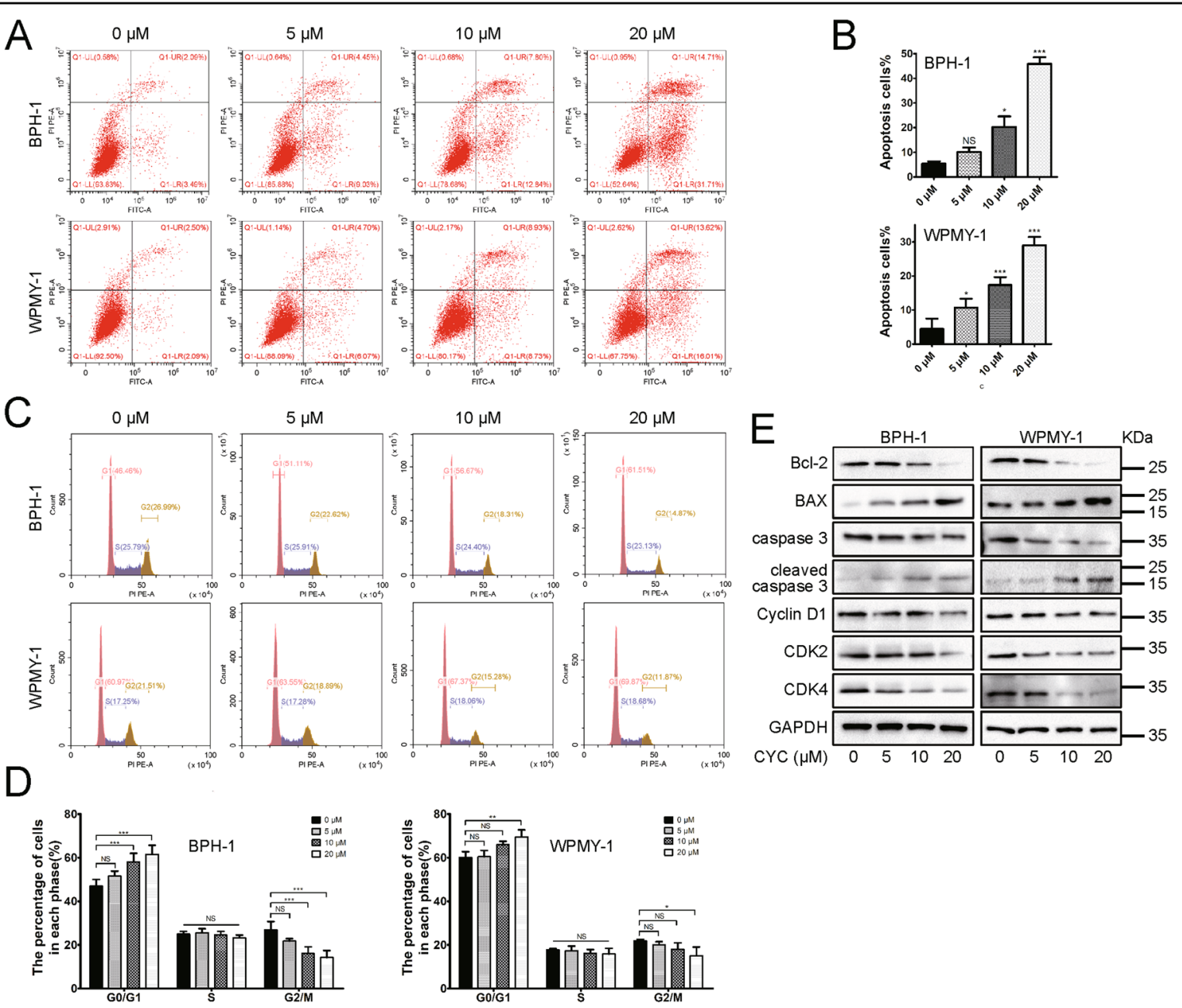

Fig. 4 Downregulation of SMO induces cell apoptosis and cell cycle arrest. A Flow cytometry analysis of the cell apoptosis in BPH-1 and WPMY1 after CYC treated for $48 \mathrm{~h}$. PI PE-A in $y$-axis stands for the fluorescence intensity of propidine iodide (PI) and FITC-A in $x$-axis stands for the fluorescence intensity of fluorescein isothiocyanate (FITC)-labeled Annexin V. Calculation area of the apoptosis rate was the percentage of Annexin V+/PI+ cells. B Statistical analysis reveals the apoptotic rate (\%) of BPH-1 and WPMY-1 after CYC treated. C Flow cytometry analysis of the cell cycle in BPH-1 and WPMY-1 after CYC treated for $48 \mathrm{~h}$. Percentages (\%) of cell populations at different stages of cell cycles were listed within the panels. D Statistical analysis of the percentages (\%) of cell populations at different stages of the cell cycles in BPH-1 and WPMY-1 after CYC treated. E Immunoblot assay of apoptosis-related and cell cycle-related protein in BPH-1 and WPMY-1 after CYC treated. NS means no significant difference, ${ }^{*} p<0.05,{ }^{* *} p<0.01$, ${ }^{* * *} p<0.001$; one-way ANOVA.

\section{CYC induced apoptosis and reduced tissue fibrosis markers in vivo}

The BPH rats had a nearly 2.5 -fold increase in Ki-67positive rate (Fig. 7A, E) and a $50 \%$ decrease in apoptosis rate detected via the terminal deoxynucleotidyl transferase dUTP nick-end labeling (TUNEL) assay (Fig. 7B, E) in rat prostate. Consistent with previous cell experiments, an increase of apoptosis rate (2.6-fold) and a decrease of $\mathrm{Ki}-67$-positive rate (45\% loss) was implied after CYC treatment. Meanwhile, the alterations of proteins associated with apoptosis and cell cycle were measured by western blotting, exhibiting upregulation of BAX and downregulation of Bcl-2, cleaved caspase-3, CDK2, CDK4, and cyclin D1 in CYC- treated rats (Fig. 7F). Furthermore, the tissue fibrosis markers $\alpha$-SMA and collagen I were analyzed in rat prostate using western blotting and immunostaining. Consistently, in the CYC-treated rats, both $\alpha$-SMA and collagen I were downregulated compared with BPH rats (Fig. 7C, D, F).

\section{Overview of SMO/GLI signaling cascades in BPH}

As shown in Fig. 8, canonical SMO/GLI signaling regulates the transcription of target genes, such as $B c l-2$, $B A X$, caspase- $3, C D K 2 / 4$, cyclin D1, $\alpha-S M A$, and collagen $I$, which subsequently modulates apoptosis, cell proliferation, cell cycle, and fibrosis through the GLIdependent transcription network in $\mathrm{BPH}^{12}$. 

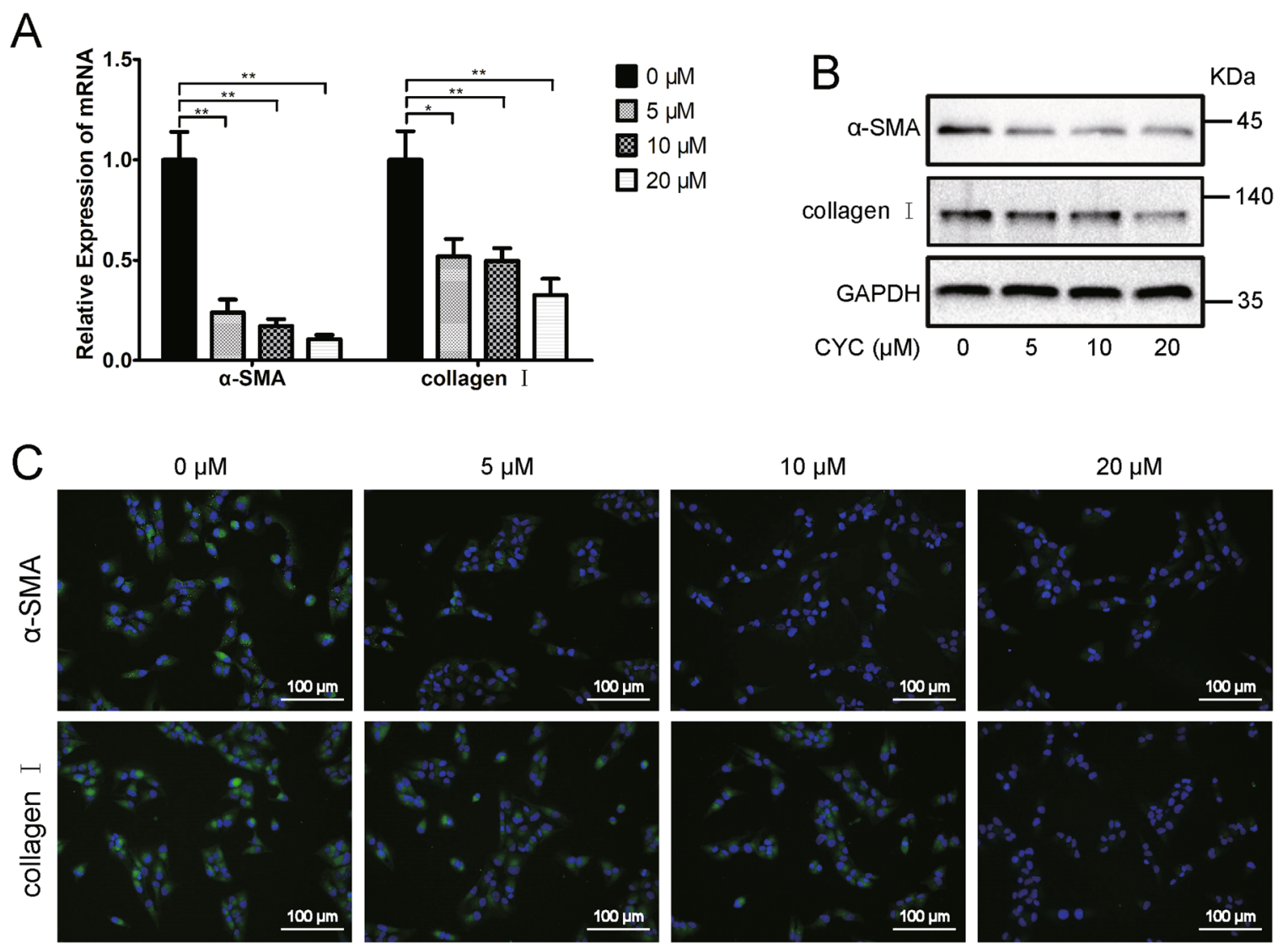

Fig. 5 Downregulation of SMO reduced fibrosis markers. A The relative mRNA level of fibrosis markers a-SMA and collagen I after CYC treated in WPMY-1. B The relative protein level of fibrosis markers a-SMA and collagen I after CYC treated in WPMY-1. C Immunofluorescence staining of a-SMA (green) and collagen I (green) in WPMY-1 after CYC treated. ${ }^{*} p<0.05,{ }^{* *} p<0.01$; one-way ANOVA. The scale bars are $100 \mu \mathrm{m}$.

\section{GLI2 and GLI3 were correlated with some clinical parameters of BPH patients}

Finally, we calculated the percentage of the positive area of these proteins on tissue microarray (TMA) of $104 \mathrm{BPH}$ patients and analyzed the correlation between the expression of these proteins and clinical parameters. As shown in Supplementary Figs. S2 and S3, SMO and GLI1-3 stained positive in both the stromal and the epithelium compartments of the human prostate. Interestingly, GLI2 was correlated positively with nocturia and negatively with free prostate-specific antigen (fPSA) (Supplementary Table S6). GLI3 was in a positive relationship with International Prostate Symptom Score (IPSS) and nocturia (Supplementary Table S6). However, we did not find any significant correlation between SMO or GLI1 with any clinical parameter (Supplementary Table S6).

\section{Discussion}

Our novel data demonstrated that SMO and GLI1-3 were localized in the stroma and the epithelium compartments of human prostate tissues and upregulated in hyperplastic prostate tissues. We also showed that SMO cascade inhibition could induce cell apoptosis, cell cycle arrest at the G0/G1 phase, and a reduction of tissue fibrosis markers both in vitro and in vivo. Our study suggested that SMO system could play a vital role in the development of $\mathrm{BPH}$.

An analysis of the human tissue-specific expression by genome-wide integration of transcriptomics ${ }^{15}$ showed that SMO and GLI1-3 were broadly expressed in the human ovary, endometrium, and other tissues, including prostate. In our current study, SMO cascade was abundantly expressed in human prostate tissues, rat ventral prostates, and cultured prostate cell lines. There are adequate experimental evidences demonstrating that prostatic stromal and epithelial cells maintain a sophisticated autocrine/paracrine type of communication ${ }^{10,16,17}$. Therefore, it is necessary for our subsequent study to determine that SMO cascade proteins can either be expressed solely in the epithelia or in both epithelia and stroma. Zhu et al. ${ }^{18}$ have reported that SMO was mainly detected in the ductal epithelia with minimal to moderate expression in the stromal cells of human fetal prostates. A 


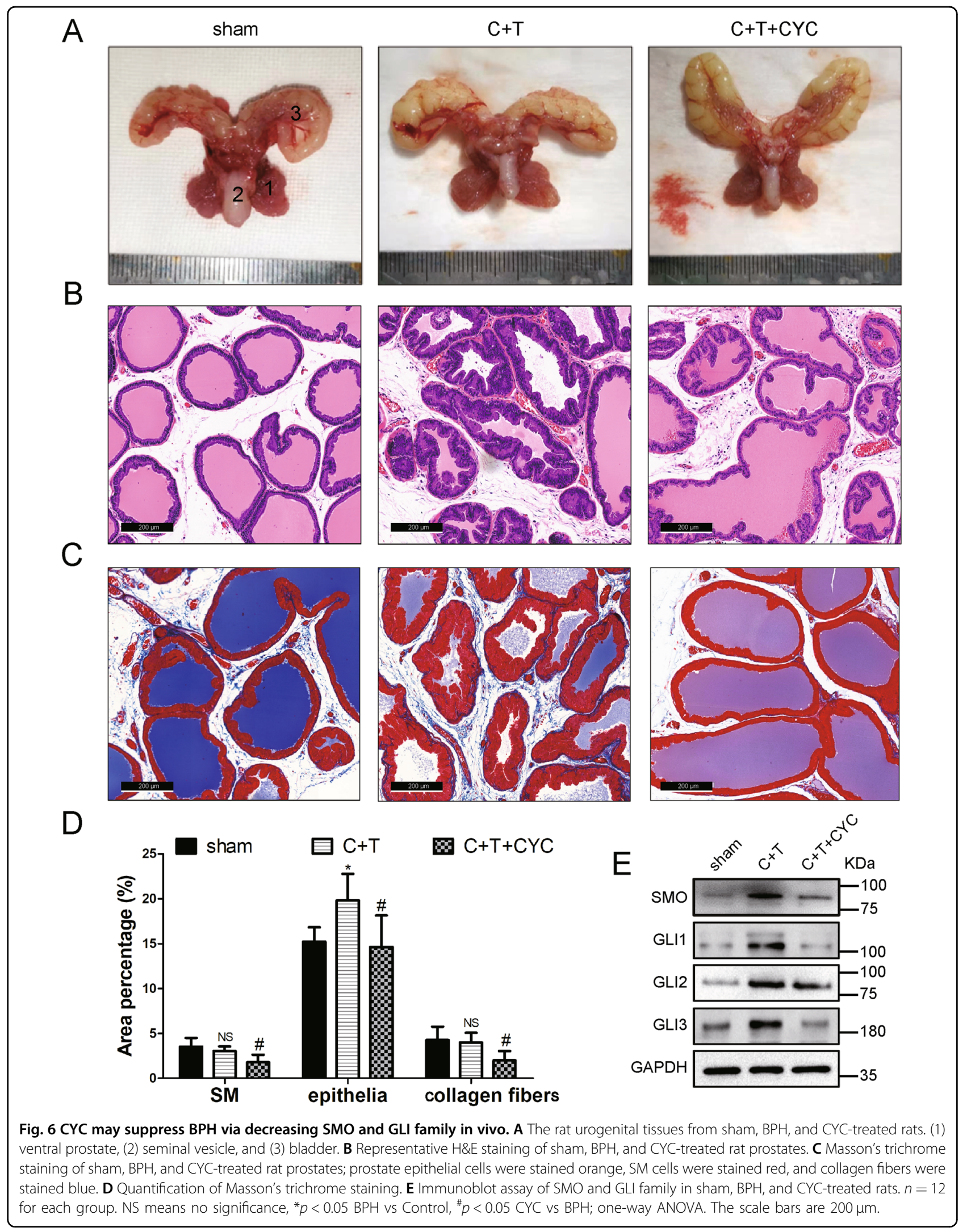




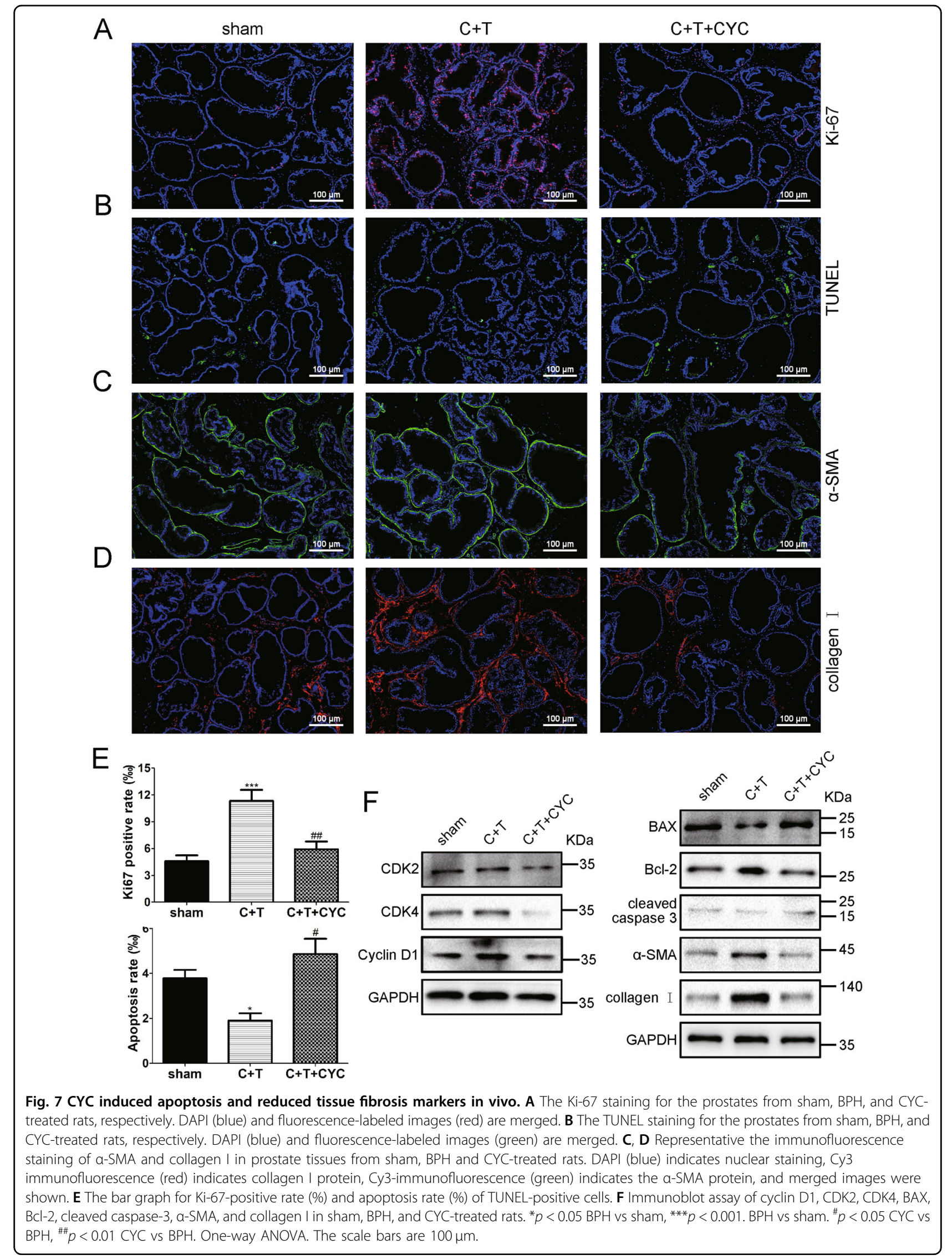




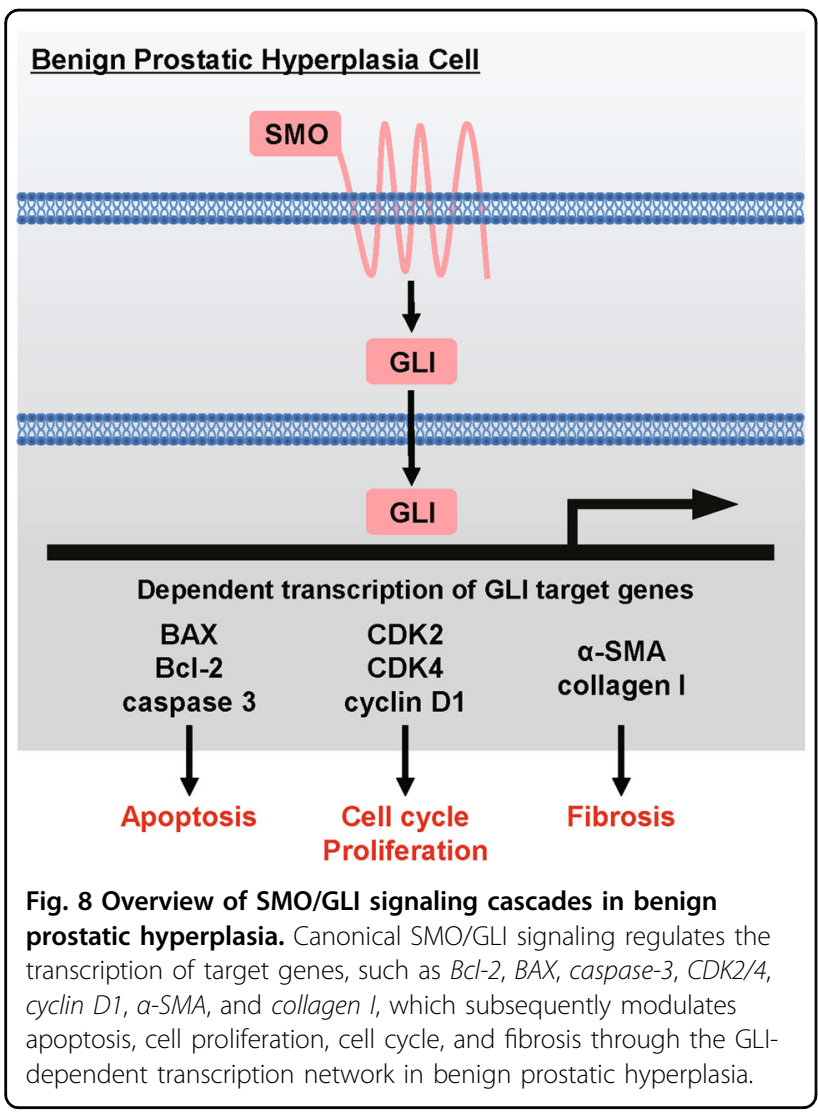

previous TMA analysis ${ }^{19}$ also showed that SMO cascade was localized in both the stromal and the epithelium compartments of adult prostate tissues, which was consistent with our current observation. The high expression of SMO cascade indicated that it could play a role in established $\mathrm{BPH}$ by modulating this autocrine/paracrine signaling pathway. Indeed, SMO, GLI2, and GLI3 were all significantly upregulated in $\mathrm{BPH}$ when compared with normal ones analyzed through two public databases (GEO and Oncomine). However, GLI1 was found to be significantly increased in $\mathrm{BPH}$ in our current study, which may attribute to a limited sample size of $\mathrm{BPH}$ or NP accessed by a public database. Besides, the sample size (eight BPH samples and eight control samples) in our current study was much larger than those public datasets of BPH. The altered expression of SMO cascade was further demonstrated via RT-PCR and western blotting. The expression levels of SMO cascade were upregulated in the human hyperplastic prostate and $\mathrm{BPH}$ rat prostate when compared with normal controls.

To explore the potential functional activities of SMO cascade involved in the progression of $\mathrm{BPH}$, two common human prostate cell lines BPH-1 and WPMY-1 were selected. SMO downregulated prostate cell model was generated using CYC treatment. CYC, a natural plantderived alkaloid, acts by inhibiting cellular response to
SMO cascade ${ }^{20,21}$. We observed that CYC could induce delayed cell proliferation, cycle arrest at the G0/G1 phase, and apoptosis for prostate cells. The percentage of apoptotic epithelial cells (45.81\%) was higher than that in stromal cells $(29.00 \%)$ at $20 \mu \mathrm{M}$ of CYC, which could be explained by the fact that prostatic epithelial cells might be more sensitive to a high dosage of CYC than stromal cells in vitro. Proteins involved in apoptosis were strongly altered, especially a high $\mathrm{BAX} / \mathrm{Bcl}-2$ ratio, which is involved in mitochondrial-dependent apoptosis ${ }^{22}$, and induce a subsequent activation of caspase- 3 and -7 down in the intrinsic apoptotic pathway ${ }^{23,24}$. Also, it was found that proteins related to cell cycle regulation, such as cyclin D1 and CDK2/4, were all reduced after CYC treatment.

Interestingly, $\mathrm{CYC}$ incubation lowered the mRNA and protein levels of fibrosis markers $\alpha$-SMA and collagen I in cultured immortalized prostate stromal cells WPMY-1. Fibrosis, as a hallmark of pathologic remodeling in many tissues, has adverse effects in many organs and leads to numerous clinical diseases ${ }^{25-28}$. During the past decades, several studies have demonstrated that fibrosis may act as a risk factor contributing to BPH/LUTS etiology ${ }^{29-32}$. Mechanistically, in response to exposure to the canonical profibrotic protein TGF- $\beta 1$ and other inflammatory proteins $^{33}$, prostate stromal fibroblasts seem to be able to differentiate into myofibroblasts and can be triggered to express fibrosis-associated collagen 1 and 3 and $\alpha$-SMA ${ }^{34}$. As CYC has a cytotoxic effect, we calculated the IC50 of CYC for the cytotoxic effect of BPH-1 and WPMY-1 cells (29.8 and $32.6 \mu \mathrm{M}$, respectively). Therefore, in this current study, we chose 5,10 , and $20 \mu \mathrm{M}$ dosages for human prostate cell lines and $10 \mathrm{mg} / \mathrm{kg}$ of CYC for in vivo intraperitoneal (i.p.) injection. Thus, CYC induced apoptosis, delayed cell growth, and reduced fibrosis markers in the prostate independent of SMO inhibition, rather than an off-target effect or a cytotoxic effect.

Over the past few decades, various types of animal models of $\mathrm{BPH}$ have been established to provide useful tools for the study of $\mathrm{BPH}$, including spontaneous $\mathrm{BPH}$ models, T-induced BPH models, xenograft models, etc. ${ }^{35}$. However, there is no animal model that could totally mimic all characteristics of human BPH. In our current study, BPH was induced in rats by castration with TP supplementation, which was a widely used, convenient, time-saving, and inexpensive model. Moreover, our TPinduced BPH model was verified with the significantly increased weight of the androgen-sensitive organs (prostate and seminal vesicle) when compared with sham rats. In line with previous observations ${ }^{36-38}$, $\mathrm{T}$ injection mainly led to a notable involution of acinar epithelium hyperplasia, such as increased acinus amount, papillary fronds protruding into the glandular cavities, and thickening of the epithelial layer with no change in the percentage of the stromal component. Interestingly, it was noticed that 
the loss of body weight in $\mathrm{BPH}$ rats seems to be due to the exogenous $\mathrm{T}$ supplementation inducing an improvement of metabolic characteristics and probably exerts its effects by a direct action on lean body mass. Meanwhile, in the CYC-treated group, BPH rats were treated with $10 \mathrm{mg} / \mathrm{kg}$ CYC (i.p.) once a day for 14 days by observing the reduction of ventral prostate weight and shrunk glands lined with a single layer of columnar epithelium to low cuboidal cells. Furthermore, both the stromal and the epithelium components were significantly reduced in the CYC-treated $\mathrm{BPH}$ rat model compared with $\mathrm{BPH}$ rats. In addition, SMO cascade proteins were upregulated in $\mathrm{BPH}$ rat prostate and downregulated with $\mathrm{CYC}$ administration. The Ki-67-positive cell rates were attenuated, whereas apoptosis rates were enhanced. The alteration of proteins involved in apoptosis and cell cycle were detected by western blotting by observing an increasing expression of BAX and a decreased expression of CDK2/4, cyclin D1, bcl-2, and cleaved caspase-3. Moreover, downregulation of SMO resulted in a suppressed status for the fibrosis markers $\alpha$-SMA and collagen I in vivo. All these changes are in parallel with our in vitro findings.

Recently, Yuan and colleagues had reported that CYC administration suppressed BPH by inhibiting epithelial and stromal cell proliferation ${ }^{39}$. In their study, only primary cultured rat prostate cells and rat models were used. No human data were available. Moreover, SMO was not determined in their study. Importantly, they lacked a more exact and scientific process of creating an agerelated BPH rat model. Their BPH rats are only $6-8$ weeks old, which is hard to find in enlarged prostate.

Finally, a TMA of 104 BPH tissues was constructed to analyze the correlation between the expression of SMO cascade proteins and corresponding clinical parameters. The GLI2 was correlated positively with nocturia and negatively with fPSA and the GLI3 was in a positive relationship with IPSS score and nocturia via a Pearson correlation analysis. The IPSS is recommended as the symptom scoring instrument that is used for the baseline assessment of symptom severity in men with LUTS $^{40}$, which includes the assessment of nocturia. Nocturia, common among older individuals with insomnia, affects people of different ages, races, and genders all over the world. The prevalence of nocturia in both men and women increased with age ${ }^{41}$. In fact, men with $\mathrm{BPH}$ more commonly present with irritative than obstructive symptoms, and the most common presenting symptom is nocturia. Our findings suggested that SMO cascade, especially GLI2 and GLI3, was in positive correlation with the progression of $\mathrm{BPH}$. However, the correlation of the SMO system with clinical data is worth further investigation.

Collectively, our present study demonstrated the expression and functional activities of SMO cascade in the prostate. SMO cascade is localized in both the stroma and the epithelium compartments of human prostate tissues and it is upregulated in hyperplastic prostate tissues. Moreover, SMO cascade appears to be involved in the development of $\mathrm{BPH}$ via modulation of imbalance of cell apoptosis, cell proliferation, and cell cycle progression, as well as the fibrosis process in vivo and in vitro. Besides, GLI2 and GLI3 are positively correlated with the progression of BPH. Thus, our data suggest that SMO cascade may represent a potential therapeutic target for the treatment of $\mathrm{BPH}$ in the future.

\section{Materials and methods \\ Public data acquiring}

Three gene expression profiling datasets (GSE6099 ${ }^{14}$, GSE3868 ${ }^{42}$, and GSE119195 ${ }^{43}$ ) were obtained from GEO (https://www.ncbi.nlm.nih.gov/geo/) of the National Center for Biotechnology Information and the mRNA expression profiles of SMO cascades between human $\mathrm{BPH}$ specimens and human NP specimens were analyzed. Meanwhile, the Oncomine database (https://www. oncomine.org/) was used for the transcriptional validation of our concerned genes.

\section{Animals and tissues}

The design of this experiment is outlined in Supplementary Fig. S1. A total of 36 specific pathogen-free grade male Sprague-Dawley rats (6 weeks old) weighing 200-250 g were used and randomly divided into three groups ( $n=12$ per group): sham group, perineal incision + corn oil (MedChemExpress, China) injection (subcutaneously (s.c.)) + dimethyl sulfoxide (DMSO) (MedChemExpress, China) administration (intraperitoneally); $\mathrm{C}+\mathrm{T}$ group, bilateral orchiectomy $+\mathrm{T}$ ( $\mathrm{T}$ propionate, Sigma-Aldrich, St. Louis, MO) (2 mg/day)/corn oil injection (s.c.) + DMSO administration (i.p.); $\mathrm{C}+\mathrm{T}+\mathrm{CYC}$ group, bilateral orchiectomy $+\mathrm{T}(2 \mathrm{mg} /$ day $) /$ corn oil injection (s.c.) + CYC (cyclopamine, MedChemExpress, China) administration $(10 \mathrm{mg} / \mathrm{kg} /$ day, i.p., according to two previous studies ${ }^{44,45}$ ). Rats were euthanized under anesthesia using a euthanasia solution containing $10 \%[\mathrm{w} / \mathrm{v}]$ chloral hydrate on day 37, and ventral prostates and seminal vesicles were harvested and weighed. The tissues were snap frozen in liquid nitrogen and stored at $-80^{\circ} \mathrm{C}$ for subsequent molecular analyses or put into $10 \%$ neutral-buffered formalin for histological examination. All surgical procedures were performed under anesthesia by i.p. injection of pentobarbital sodium $(35 \mathrm{mg} / \mathrm{kg}$; Abbott Laboratory, Chicago, IL). We are blinded to the group allocation when assessing the in vivo effect of CYC on rat prostates. Animal experiments were conducted at the Animal Center of Zhongnan Hospital of Wuhan University and all animal protocols were approved by the Medical Ethics Committee for Experimental animals of Zhongnan Hospital of Wuhan University. 
Eight prostate samples from young brain-dead men (mean age, 28.2 \pm 4.4 years old) undergoing organ donation were obtained as controls. A total of 104 specimens of BPH samples with clinical data were obtained from the patients who underwent transurethral resection prostate at the Department of Urology, Zhongnan Hospital of Wuhan University. Postoperative prostate pathology examination revealed $\mathrm{BPH}$. Prostate tissues were divided into two strips and were, respectively, stored in liquid nitrogen for PCR and western blotting analysis and stored in 10\% neutral-buffered formalin for immunofluorescence microscopy and the construction of TMA. Collection and treatment of all human specimens were carried out in accordance with the approved guidelines of the Ethics Committee at Zhongnan Hospital of Wuhan University.

\section{The construction and immunohistochemistry analysis of TMA}

The summarized clinical data of $104 \mathrm{BPH}$ patients was present in Supplementary Table S1. The paraffinembedded specimens were sliced followed by hematoxylin and eosin (H\&E) staining. The representative areas of the H\&E staining sections were evaluated and confirmed by a senior pathologist. A TMA marker was designed by using $1.5 \mathrm{~mm}$ tissue core in each case. Finally, TMA contained $16 \times 10$ tissue cores for all BPH specimens in each were obtained and then sliced continuously into $4-\mu \mathrm{m}$-thick sections. Briefly, paraffin sections were deparaffinized first, then antigen retrieval was performed in citrate buffer (pH 6.0), and endogenous peroxidase activity was blocked in $0.3 \% \mathrm{H}_{2} \mathrm{O}_{2}$. Subsequently, all slides were incubated with primary and secondary antibodies (listed in Supplementary Tables S2 and S3) until visualization by peroxidase and 3, 3'-diaminobenzidine tetrahydrochloride. All the stained sections were imaged using Olympus-DP72 light microscope (Olympus, Japan). The expression of SMO and GLI1-3 in the prostate tissues from the TMA was blindly quantified by two pathologists. The percentage of protein-positive area was measured by Image J.

\section{Cell culture}

Human benign prostatic enlargement epithelial cell line BPH-1 (Cat. \#BNCC339850) was purchased from the Procell Co., Ltd. in Wuhan, China. Identification of the cell lines was performed at the China Center for Type Culture Collection in Wuhan, China. SV40 large-T antigen-immortalized stromal cell line WPMY-1 (Cat. \#GNHu36) was purchased from the Stem Cell Bank, Chinese Academy of Sciences in Shanghai, China. The BPH-1 cells were cultured in RPMI-1640 medium (Gibco, China) containing 10\% fetal bovine serum (FBS) (Gibco, Australia). The WPMY-1 cells were cultured in Dulbecco's modified Eagle's medium (Gibco, China) containing $1 \%$ penicillin G sodium/streptomycin sulfate and 5\% FBS.
All the cell lines were recently authenticated and were cultured in a humidified atmosphere consisting of $95 \%$ air and $5 \% \mathrm{CO}_{2}$ at $37^{\circ} \mathrm{C}$.

\section{Flow cytometry analysis}

For cell cycle analysis, BPH-1 and WPMY- 1 cells $(1 \times$ $10^{6}$ cells) were harvested, washed with phosphatebuffered saline (PBS), and then centrifuged. Pellets were resuspended with $1 \mathrm{ml}$ DNA staining solution, which contained $50 \mu \mathrm{g} / \mathrm{ml}$ propidium iodide and $0.1 \mathrm{mg} / \mathrm{ml}$ RNaseA, and $10 \mu \mathrm{l}$ permeabilization solution (Multisciences, China). The DNA content distribution was analyzed by flow cytometry analysis (Beckman, Cat. \#FC500, USA) after incubation in the dark at $37^{\circ} \mathrm{C}$ for $30 \mathrm{~min}$. For cell apoptosis analysis, fluorescein isothiocyanate (FITC) Annexin V Apoptosis Detection Kit I (BD Biosciences, USA) was used. BPH-1 and WPMY-1 cells $\left(1 \times 10^{6}\right.$ cells $)$ were harvested and then stained with FITC Annexin V Apoptosis Detection Kit I according to the manufacturer's instruction.

\section{CCK-8 assay for cell viability}

The viability of cells was examined by CCK- 8 (MedChemExpress, China) assay. Briefly, the neurons ( 5000 cells/well) were seeded in poly-L-lysine-coated 96-well plates and subjected to various treatments as described above. CCK- 8 solution $(10 \mu \mathrm{l} / 100 \mu \mathrm{l})$ was added to each culture well, and neurons were incubated for $2 \mathrm{~h}$ at $37^{\circ} \mathrm{C}$. Finally, we measured the absorbance at $450 \mathrm{~nm}$ with a microplate reader (Thermo Labsystems, Vantaa, Finland).

\section{Cell immunofluorescence staining}

For cell immunofluorescence microscopy, cells were cultured as aforementioned, followed by seeding on $12 \mathrm{~mm}$ coverslips and washing by ice-cold (PBS ( $\mathrm{pH}=$ 7.4). The coverslips were then fixed with $4 \%$ paraformaldehyde (PFA) for $30 \mathrm{~min}$, followed by $0.1 \%$ Triton $\mathrm{X}-100$ incubation, and then blocked in goat serum for $30 \mathrm{~min}$ at room temperature. Afterward, they were incubated with primary antibody (listed in Supplementary Table S2) at room temperature for $2 \mathrm{~h}$, washed with PBS, and incubated with Cy3- or FITC-labeled secondary antibody (listed in Supplementary Table S3) for $1 \mathrm{~h}$. Nuclei were labeled with DAPI (4',6-diamidino-2-phenylindole) $(2 \mu \mathrm{g} / \mathrm{ml})$. Visualization was done with a laser scanning confocal microscope (Olympus, Tokyo, Japan).

\section{RNA isolation, reverse transcription, and quantitative real- time PCR (qRT-PCR) analysis}

Total RNA was isolated from frozen tissues and cell lines using Trizol reagent (Invitrogen, Carlsbad, CA, USA) according to the manufacturer's instructions and quantitated at $260 / 280 \mathrm{~nm}$ using a NanoPhotometer spectrophotometer (IMPLEN, Westlake Village, CA, USA). Two 
micrograms of total RNA was reverse transcribed to complementary DNA via the SuperScript II First-Strand Synthesis System according to the manufacturer (Invitrogen, Carlsbad, CA, USA). QRT-PCR was performed to determine the level of mRNA expression of a gene of interest based on SYBR Green using a Bio-Rad (Hercules, CA, USA) CFX96 system. The expression levels of genes were normalized to the expression of glyceraldehyde 3phosphate dehydrogenase (GAPDH) mRNA and compared by $2^{-\Delta \Delta C T}$ method. Primer sequences are listed in Supplementary Table S4. Values were normalized for amplified GAPDH alleles.

\section{Western blotting analysis}

Tissues and cell lines were lysed and ultrasonicated in RIPA reagent containing protease inhibitor and phosphatase inhibitor (Sigma-Aldrich, St. Louis, MO, USA) on ice for $30 \mathrm{~min}$. The supernatant was collected after centrifugation at $14,000 \times g$ for $10 \mathrm{~min}$ at $4{ }^{\circ} \mathrm{C}$. Then, the protein concentration was measured by bicinchoninic acid assay. Protein extracts were isolated by sodium dodecyl sulfatepolyacrylamide gel electrophoresis and then transferred to a polyvinylidene fluoride membrane (Millipore, Billerica, MA, USA) using a Bio-Rad wet transfer system. The membrane was then blocked in Tris-buffered saline with $0.05 \%$ Tween-20 buffer containing 5\% skim milk, and incubated sequentially with primary and secondary antibodies (listed in Supplementary Tables S2 and S3). An Enhanced Chemiluminescence Kit (Thermo Fisher Scientific, Waltham, MA, USA) was used to expose the bands.

\section{H\&E staining}

Prostate paraffin sections $(5 \mu \mathrm{m})$ were deparaffinized in xylene for $3 \times 10 \mathrm{~min}$, rehydrated in descending concentrations of ethanol $(100,96,80$, and $70 \%)$, and $\mathrm{H}_{2} \mathrm{O}$. The sections were then stained in $10 \%$ hematoxylin (Sigma-Aldrich, St. Louis, MO, USA) for $7 \mathrm{~min}$, followed by washing under the tap water for $10 \mathrm{~min}$ to reveal the nuclei. Afterward, the sections were stained in $1 \%$ eosin (Sigma-Aldrich, St. Louis, MO, USA) containing 0.2\% glacial acetic acid for $5 \mathrm{~min}$. After staining, the sections were washed with tap water, dehydrated in increasing grades of ethanol $(70,80,96$, and $100 \%)$, and cleared in xylene for $3 \times 10 \mathrm{~min}$. The sections were imaged by an Olympus-DP72 light microscope (Olympus, Japan).

\section{Masson's trichrome staining}

As previously described ${ }^{46}$, prostate tissues were embedded into paraffin after being fixed in $10 \%$ formalin for $24-36 \mathrm{~h}$ and cut into $5 \mu \mathrm{m}$ sections. Then, the sections were stained using Masson's trichrome staining. Staining was detected by light microscopy. Prostatic smooth muscle (SM) cells, collagen fibers, and epithelial cells were stained red, blue, and orange, respectively. In each sample, we analyzed three areas randomly under magnification $(\times 200)$. The area percentage of SM, collagen fibers, and glandular epithelium were quantitated with Image Pro Plus 5.0.

\section{TUNEL assay}

Rat prostate tissues were fixed in 4\% PFA, embedded in paraffin, and then digested with proteinase $\mathrm{K}$ for $20 \mathrm{~min}$. The sections were then incubated with a fluorometric DNA Fragmentation Detection Kit (PromoCell, Heidelberg, Germany) according to the manufacturer's instructions. Nuclei were labeled with DAPI. Visualization was done with a Laser Scanning Confocal Microscope (Olympus, Tokyo, Japan). DAPI and fluorescence-labeled images were merged and TUNEL-positive apoptotic cells in the merged images were quantified by the counting of positively stained cells.

\section{Tissue immunofluorescent staining}

Tissues were sectioned in $10-\mu \mathrm{m}$-thick slices and thawed, mounted onto glass slides using a cryostat (Leica CM 1850, Wetzlar, Germany), air-dried, and fixed for $10 \mathrm{~min}$ in icecold acetone. Slides were washed in PBS and incubated for $2 \mathrm{~h}$ in a mixture of PBS supplemented with $0.2 \%$ Triton X100 and $0.1 \%$ bovine serum albumin, followed by incubation overnight with the primary antibodies (listed in Supplementary Table S2). The secondary antibodies (listed in Supplementary Table S3) employed to visualize the localization of SMO and GLI1-3 were Cy3-conjugated goat antirabbit IgG (1:1000). DAPI was used for staining the nucleus. Visualization was done with a Laser Scanning Confocal Microscope (Olympus, Tokyo, Japan).

\section{Statistical analysis}

All analyses were performed at least three times and represented data from three individual experiments. The data values were expressed as the means \pm standard deviation (SD). Statistical analysis was performed using either the Student's $t$ test (two groups compared) or oneway analysis of variance and Tukey's post tests with SPSS 20.0 (multiple means compared). Statistical significance was considered as a $p$ value $<0.05$.

\section{Acknowledgements}

We thank the staff at Zhongnan Hospital of Wuhan University for their help.

\section{Author details}

'Department of Urology, Zhongnan Hospital of Wuhan University, Wuhan, China. ${ }^{2}$ Department of Rehabilitation, Zhongnan Hospital of Wuhan University, Wuhan, China. ${ }^{3}$ Department of Surgery and Biomedical Sciences, Cooper Medical School of Rowan University, Camden, NJ, USA

\section{Funding}

This study was supported in part by the National Natural Science Foundation of China (Nos. 82070780 and 81770757 ).

\section{Ethical approval}

Collection and treatment of all human specimens were carried out in accordance with the approved guidelines of the Ethics Committee at Zhongnan Hospital of Wuhan University. All animal protocols were approved 
by the Medical Ethics Committee for Experimental animals of Zhongnan Hospital of Wuhan University.

\section{Conflict of interest}

The authors declare no competing interests.

\section{Publisher's note}

Springer Nature remains neutral with regard to jurisdictional claims in published maps and institutional affiliations.

Supplementary information The online version contains supplementary material available at https://doi.org/10.1038/s41420-021-00501-4.

Received: 22 March 2021 Revised: 10 April 2021 Accepted: 28 April 2021 Published online: 18 May 2021

\section{References}

1. Gratzke, C. et al. EAU Guidelines on the Assessment of non-neurogenic male lower urinary tract symptoms including benign prostatic obstruction. Eur. Urol. 67, 1099-1109 (2015).

2. Rosen, R. C., Giuliano, F. \& Carson, C. C. Sexual dysfunction and lower urinary tract symptoms (LUTS) associated with benign prostatic hyperplasia (BPH). Eur. Urol. 47, 824-837 (2005).

3. Roehrborn, C. G. Benign prostatic hyperplasia: an overview. Rev. Urol. 7(Suppl. 9), S3-S14 (2005).

4. Wang, Y., Lu, P., Zhao, D. \& Sheng, J. Targeting the hedgehog signaling pathway for cardiac repair and regeneration. Herz 42, 662-668 (2017).

5. Bond, C., Tang, Y. \& Podlasek, C. A. Neural influences on sonic hedgehog and apoptosis in the rat penis. Biol. Reprod. 78, 947-956 (2008).

6. Haraguchi, R. et al. Recent insights into long bone development: central role of hedgehog signaling pathway in regulating growth plate. Int. J. Mol. Sci. 20, https://doi.org/10.3390/ijms20235840 (2019).

7. Han, L. et al. Osr1 functions downstream of Hedgehog pathway to regulate foregut development. Dev. Biol. 427, 72-83 (2017).

8. Brennen, W. N. \& Isaacs, J. T. Mesenchymal stem cells and the embryonic reawakening theory of BPH. Nat. Rev. Urol. 15, 703-715 (2018).

9. $\mathrm{Yu}, \mathrm{M}$. et al. Sonic hedgehog-responsive genes in the fetal prostate. J. Biol. Chem. 284, 5620-5629 (2009).

10. Shaw, A. \& Bushman, W. Hedgehog signaling in the prostate. J. Urol. 177 832-838 (2007).

11. Infante, P., Alfonsi, R., Botta, B., Mori, M. \& Di Marcotullio, L. Targeting GLI factors to inhibit the Hedgehog pathway. Trends Pharmacol. Sci. 36, 547-558 (2015).

12. Katoh, M. Genomic testing, tumor microenvironment and targeted therapy of Hedgehog-related human cancers. Clin. Sci. 133, 953-970 (2019).

13. Pandolfi, S. \& Stecca, B. Cooperative integration between HEDGEHOG-GLI signalling and other oncogenic pathways: implications for cancer therapy. Expert Rev. Mol. Med. 17, e5 (2015).

14. Tomlins, S. A. et al. Integrative molecular concept modeling of prostate cancer progression. Nat. Genet. 39, 41-51 (2007).

15. Fagerberg, L. et al. Analysis of the human tissue-specific expression by genome-wide integration of transcriptomics and antibody-based proteomics. Mol. Cell. Proteom. 13, 397-406 (2014).

16. Wang, N. et al. Regulation of prostate development and benign prostatic hyperplasia by autocrine cholinergic signaling via maintaining the epithelial progenitor cells in proliferating status. Stem Cell Rep. 6, 668-678 (2016).

17. La Vignera, S., Condorelli, R. A., Russo, G. I., Morgia, G. \& Calogero, A. E. Endocrine control of benign prostatic hyperplasia. Andrology 4, 404-411 (2016).

18. Zhu, G. et al. Sonic and desert hedgehog signaling in human fetal prostate development. Prostate 67, 674-684 (2007).

19. Gonnissen, A. et al. Tissue microarray analysis indicates hedgehog signaling as a potential prognostic factor in intermediate-risk prostate cancer. BMC Cancer 17, 634 (2017).

20. Ma, H., Li, H. Q. \& Zhang, X. Cyclopamine, a naturally occurring alkaloid, and its analogues may find wide applications in cancer therapy. Curr. Top. Med. Chem. 13, 2208-2215 (2013).
21. Taipale, J. et al. Effects of oncogenic mutations in Smoothened and Patched can be reversed by cyclopamine. Nature 406, 1005-1009 (2000).

22. $\mathrm{Hu}, \mathrm{G}$. et al. Stomatin-like protein 2 inhibits cisplatin-induced apoptosis through MEK/ERK signaling and the mitochondrial apoptosis pathway in cervical cancer cells. Cancer Sci. 109, 1357-1368 (2018).

23. Salakou, S. et al. Increased Bax/BCl-2 ratio up-regulates caspase- 3 and increases apoptosis in the thymus of patients with myasthenia gravis. In Vivo 21, 123-132 (2007).

24. Ma, Y. et al. Regulation of intrinsic and extrinsic apoptotic pathways in osteosarcoma cells following oleandrin treatment. Int. J. Mol. Sci. 17, https:// doi.org/10.3390/ijms17111950, (2016).

25. Rockey, D. C., Bell, P. D. \& Hill, J. A. Fibrosis-a common pathway to organ injury and failure. N. Engl. J. Med. 372, 1138-1149 (2015).

26. Weiskirchen, R., Weiskirchen, S. \& Tacke, F. Organ and tissue fibrosis: molecular signals, cellular mechanisms and translational implications. Mol. Asp. Med. 65, 2-15 (2019).

27. Gharaee-Kermani, M., Hu, B., Phan, S. H. \& Gyetko, M. R. Recent advances in molecular targets and treatment of idiopathic pulmonary fibrosis: focus on TGFbeta signaling and the myofibroblast. Curr. Med. Chem. 16, 1400-1417 (2009).

28. Novo, E., di Bonzo, L. V., Cannito, S., Colombatto, S. \& Parola, M. Hepatic myofibroblasts: a heterogeneous population of multifunctional cells in liver fibrogenesis. Int. J. Biochem. Cell Biol. 41, 2089-2093 (2009).

29. Ma, J. et al. Prostatic fibrosis is associated with lower urinary tract symptoms. J. Urol. 188, 1375-1381 (2012).

30. Djavan, B. et al. Elastin gene expression in benign prostatic hyperplasia. Prostate 40, 242-247 (1999).

31. Cantiello, F. et al. Periurethral fibrosis secondary to prostatic inflammation causing lower urinary tract symptoms: a prospective cohort study. Urology 81, 1018-1023 (2013).

32. Bauman, T. M. et al. Characterization of fibrillar collagens and extracellular matrix of glandular benign prostatic hyperplasia nodules. PLOS ONE 9, e109102 (2014).

33. Gharaee-Kermani, M. et al. CXC-type chemokines promote myofibroblast phenoconversion and prostatic fibrosis. PLOS ONE 7, e49278 (2012).

34. Rodriguez-Nieves, J. A. \& Macoska, J. A. Prostatic fibrosis, lower urinary tract symptoms, and BPH. Nat. Rev. Urol. 10, 546-550 (2013).

35. Zhang, J. et al. Animal models of benign prostatic hyperplasia. Prostate Cancer Prostatic Dis. https://doi.org/10.1038/s41391-020-00277-1 (2020).

36. Al-Trad, B., Aljabali, A., Al Zoubi, M., Shehab, M. \& Omari, S. Effect of gold nanoparticles treatment on the testosterone-induced benign prostatic hyperplasia in rats. Int. J. Nanomed. 14, 3145-3154 (2019).

37. Chen, $P$. et al. Testosterone regulates myosin ॥ isoforms expression and functional activity in the rat prostate. Prostate 78, 1283-1298 (2018).

38. Zhang, $X$. et al. Testosterone regulates smooth muscle contractile pathways in the rat prostate: emphasis on PDE5 signaling. Am. J. Physiol. Endocrinol. Metab. 302, E243-E253 (2012).

39. Yuan, Y. F. et al. Cyclopamine functions as a suppressor of benign prostatic hyperplasia by inhibiting epithelial and stromal cell proliferation via suppression of the Hedgehog signaling pathway. Int. J. Mol. Med. 46, 311-319 (2020).

40. McVary, K. T. et al. Update on AUA guideline on the management of benign prostatic hyperplasia. J. Urol. 185, 1793-1803 (2011).

41. Bosch, J. L. \& Weiss, J. P. The prevalence and causes of nocturia. J. Urol. 184 440-446 (2010).

42. Nanni, S. et al. Epithelial-restricted gene profile of primary cultures from human prostate tumors: a molecular approach to predict clinical behavior of prostate cancer. Mol. Cancer Res. 4, 79-92 (2006).

43. Xiao, $H$. et al. Identification and functional activity of matrix-remodeling associated 5 (MXRA5) in benign hyperplastic prostate. Aging 12, 8605-8621 (2020).

44. $\mathrm{Yu}, \mathrm{P}$. et al. Resveratrol pretreatment decreases ischemic injury and improves neurological function via sonic hedgehog signaling after stroke in rats. Mol. Neurobiol. 54, 212-226 (2017).

45. Pratap, A. et al. Cyclopamine attenuates acute warm ischemia reperfusion injury in cholestatic rat liver: hope for marginal livers. Mol. Pharmaceutics $\mathbf{8}$ 958-968 (2011)

46. Zhang, W. et al. Upregulation of phosphodiesterase type 5 in the hyperplastic prostate. Sci. Rep. 5, 17888 (2015). 